\title{
Modern treatment of pulmonary embolism
}

\author{
S.Z. Goldhaber
}

\begin{abstract}
Modern treatment of pulmonary embolism. S.Z. Goldhaber. (C) ERS Journals Ltd 2002. ABSTRACT: Modern treatment of acute pulmonary embolism requires rapid and accurate diagnosis followed by risk stratification to devise an optimal management strategy. Patients at low risk have good outcomes simply with intensive anticoagulation treatment. Higher-risk patients may require more aggressive intervention with thrombolysis or embolectomy.

Clinical risk factors for an adverse outcome include increasing age, cancer, congestive heart failure, systemic arterial hypotension, chronic obstructive pulmonary disease and right ventricular dysfunction. A promising approach is the Geneva Prognostic Score, which is based upon a rapid clinical assessment.

On physical examination, signs of right ventricular failure, including distended jugular veins and a right-sided $S_{3}$ gallop, should be looked for. The electrocardiogram may show evidence of right ventricular strain with a new right bundle branch block or $T$ wave inversion in leads V1-V4. The troponin level may be elevated as a marker of cardiac injury and right ventricular microinfarction, even in the absence of coronary artery disease. The most useful imaging marker of high risk is the presence of moderate or severe right ventricular dilatation and hypokinesis on the echocardiogram, especially with progressively worsening right ventricular function despite intensive anticoagulation treatment.

Patients at high risk should be considered for thrombolytic therapy or embolectomy rather than management with anticoagulation therapy alone. Special care must be taken to avoid thrombolytic therapy among patients who might be susceptible to intracranial haemorrhage. Intracranial haemorrhage reached a surprisingly high rate of $3.0 \%$ in the International Cooperative Pulmonary Embolism Registry of 2,454 prospectively evaluated acute pulmonary embolism patients at 52 hospitals in seven countries. An alternative approach to patients at high risk is a catheter-based or open surgical embolectomy. It is crucial to refer these patients as quickly as possible, rather than delaying intervention until cardiogenic shock has ensued. Fortunately the current tools for risk stratification provide an "early window" for prognostication and can help the coordination of a definitive treatment plan with optimal results.
\end{abstract}

Eur Respir J 2002; 19: Suppl. 35, 22s-27s.

\author{
Correspondence: S.Z. Goldhaber \\ Cardiovascular Division \\ Brigham and Women's Hospital \\ Harvard Medical School \\ Boston \\ MA 02115 \\ USA \\ Fax: 16172645144 \\ Email: sgoldhaber@partners.org
}

Keywords: Deep vein thrombosis embolectomy

pulmonary embolism

thrombolysis

Received: August 102001

Accepted August 102001
Modern treatment of pulmonary embolism (PE) relies upon rapid identification, risk stratification and anticoagulation treatment as the foundations of therapy. Patients identified as at high risk of adverse outcomes often benefit from early intervention with thrombolytic therapy or embolectomy. Depending upon the severity and specific problems related to the acute PE, special expertise might be required from radiologists, vascular medicine specialists, cardiologists, pulmonologists, critical care intensivists, interventional angiographers or cardiac surgeons. Optimal management requires a coordinated interdisciplinary approach that follows algorithms established by consensus at individual hospitals.

\section{Clinical outcome}

The International Cooperative Pulmonary Embolism Registry (ICOPER) enrolled 2,454 consecutive $\mathrm{PE}$ patients from 52 participating hospitals in seven countries. The aims were to establish a 3-month allcause mortality rate and identify factors associated with death [1].

The 3-month follow-up was completed in 98\% of patients. The all-cause mortality rate was $11.4 \%$ during the first 2 weeks after diagnosis and $17.4 \%$ at 3 months. After exclusion of patients in whom PE was first discovered at autopsy, the mortality rate was $15.3 \%$. Importantly, most patients who died had succumbed to PE, and not to other comorbidities such as cancer. Specifically, regarding the most common causes of death, $45 \%$ of deaths were ascribed to $\mathrm{PE}, 18 \%$ were due to cancer, $12 \%$ were sudden cardiac deaths (which undoubtedly included some undiagnosed PEs) and 12\% were considered due to respiratory failure, which may also have included some deaths due to PE. Nonfatal recurrent PE occurred in $4 \%$ of patients.

Age $>70$ yrs increased the likelihood of death by $60 \%$. Six other risk factors independently increased the likelihood of mortality two- to three-fold (table 1): 
Table 1.-Risk factors for mortality after pulmonary embolism in the International Cooperative Pulmonary Embolism Registry: a multivariate analysis

\begin{tabular}{lc}
\hline Variable & $\begin{array}{c}\text { Hazard ratio } \\
(95 \% \mathrm{CI})\end{array}$ \\
\hline Age $>70$ yrs & $1.6(1.1-2.3)$ \\
Cancer & $2.3(1.5-3.5)$ \\
Clinical congestive heart failure & $2.4(1.5-3.7)$ \\
Chronic obstructive pulmonary disease & $1.8(1.2-2.7)$ \\
Systolic blood pressure $<90 \mathrm{mmHg}$ & $2.9(1.7-5.0)$ \\
Respiratory frequency $<20$ breathe $\cdot \min ^{-1}$ & $2.0(1.2-3.2)$ \\
Right ventricular hypokinesis & $2.0(1.3-2.9)$ \\
\hline
\end{tabular}

The final model included 815 patients. CI: confidence interval. (From [1].)

cancer, clinical congestive heart failure, chronic obstructive pulmonary disease, systemic arterial hypotension with a systolic blood pressure of $<90 \mathrm{mmHg}$, tachypnoea (defined as $>20$ breaths $\cdot \mathrm{min}^{-1}$ ) and right ventricular hypokinesis on the echocardiogram, an especially useful sign for identifying high-risk patients who might be suitable for aggressive interventions such as thrombolytic therapy or embolectomy.

In Japan, a recently completed PE registry showed remarkable similarities to the ICOPER [2]. The inhospital mortality rate was $14 \%$ among the $533 \mathrm{PE}$ patients enrolled in the registry. Predictors of mortality included male sex, cardiogenic shock, cancer and prolonged immobilization.

\section{Risk stratification}

In patients with massive PE, right ventricular dysfunction can be identified at the bedside. Findings include a left parasternal heave, distended jugular veins, and a systolic murmur of tricuspid regurgitation that increases with inspiration and is best heard at the left lower sternal border. The electrocardiogram may demonstrate a new right bundle branch block or other evidence of right ventricular strain, such as inverted $\mathrm{T}$ waves in leads V1-V4.

The Geneva Risk Score was developed using data from $296 \mathrm{PE}$ patients [3]. It predicts the risk of major adverse outcomes, defined as death, recurrent PE and major bleeding. Six prognostic variables are considered. Patients receive a point for each variable and an estimate of the likelihood of an adverse outcome (tables 2 and 3). Cancer and hypotension receive the greatest weight, followed by congestive heart failure, prior venous thromboembolism, hypoxaemia and evidence of acute deep vein thrombosis (DVT) on ultrasound examination.

Right ventricular dysfunction has been reliably established as a predictor of increased likelihood of death from PE. Identification of moderate or severe right ventricular dysfunction is usually carried out qualitatively and is ordinarily readily apparent to observers with only modest experience. The most commonly employed quantitative standards are: 1) a right ventricular to left ventricular end-diastolic diameter ratio of $>1$ in the apical four-chamber
Table 2.-The Bounameaux pulmonary embolism point score

\begin{tabular}{lc}
\hline Variable & Point score \\
\hline Cancer & +2 \\
Heart failure & +1 \\
Prior DVT & +1 \\
Hypotension & +2 \\
Hypoxaemia & +1 \\
DVT on ultrasound & +1 \\
\hline
\end{tabular}

DVT: deep vein thrombosis. (From [3].)

view; 2) a right ventricular end-diastolic diameter of $>30 \mathrm{~mm}$; and 3) paradoxical right ventricular septal systolic motion.

Among 209 consecutive PE patients, 65 (31\%) presented with the combination of normal systemic arterial pressure and echocardiographic evidence of right ventricular dysfunction. Of this group, six (10\%) developed cardiogenic shock within $24 \mathrm{~h}$ of diagnosis and three $(5 \%)$ died during the initial hospitalization. Conversely, none of the 97 normotensive patients with normal right ventricular function on echocardiography suffered PE-related mortality [4].

When Doppler echocardiography gives an estimated pulmonary artery systolic pressure of $>50 \mathrm{mmHg}$ at the time of PE diagnosis, chronic pulmonary hypertension is likely to ensue. Such patients are less likely to survive during the ensuing 5 yrs than those whose pulmonary arterial pressure and right ventricular function normalize during the first 5-6 weeks after the acute PE [4].

Right ventricular microinfarction can result from the right ventricular pressure overload caused by acute PE and can be diagnosed by means of elevated troponin levels. Such patients may have no underlying atherosclerosis of the right coronary artery. Troponin level elevation in acute PE is associated with right ventricular dysfunction [5]. Furthermore, troponin level elevations help identify PE patients with an otherwise poor prognosis in whom aggressive intervention may be warranted. In a prospective study of $56 \mathrm{PE}$ patients, those with elevated troponin levels were more likely to die, suffer cardiogenic shock, require inotropic agents and need mechanical ventilation [6]. The mortality rate from $\mathrm{PE}$ was $44 \%$ in troponin-positive patients (i.e. those with elevated

Table 3. - The Bounameaux adverse outcome score

\begin{tabular}{lccc}
\hline \multirow{2}{*}{ Points } & \multicolumn{3}{c}{ Patients } \\
\cline { 2 - 4 } & $\mathrm{n}$ & cumulative \% & adverse outcome \% (n) \\
\hline 0 & 52 & 19.4 & $0(0)$ \\
1 & 79 & 48.9 & $2.5(2)$ \\
2 & 49 & 67.2 & $4.1(2)$ \\
3 & 56 & 88.1 & $17.8(10)$ \\
4 & 22 & 96.3 & $27.3(6)$ \\
5 & 7 & 98.9 & $57.1(4)$ \\
6 & 3 & 100 & $100(3)$ \\
\hline
\end{tabular}

(From [3].) 
troponin levels), compared with $3 \%$ in troponinnegative patients (i.e. those without elevated troponin levels). Interestingly, coronary angiography revealed obstructive coronary artery disease equally distributed between troponin-positive and troponin-negative patients.

\section{Thrombolytic therapy and embolectomy}

Thrombolytic therapy and embolectomy should be considered after risk stratification in the following four scenarios: 1) haemodynamic instability; 2) moderate or severe right ventricular dysfunction; 3) elevation of the troponin level; and 4) a high Geneva Risk Score. Both thrombolytic therapy and embolectomy are enjoying a renaissance because of improvements in pharmacological understanding and the timing of referrals for aggressive intervention. It is imperative that attempts to intervene in high-risk patients are made as early as possible, prior to the onset of cardiogenic shock. The "watch and wait" approach is usually not warranted. When thrombolytic therapy is administered, a high concentration and short infusion of the fibrinolytic agent should be prescribed, rather than a prolonged infusion of a low-to-moderate concentration of drug. Embolectomy should be considered for those patients with contraindications to thrombolytic therapy.

\section{Thrombolytic therapy: overview}

The ideal thrombolytic agent has not yet been developed. Such a drug would rarely cause major bleeding and could be administered at low cost with precisely defined indications. Currently, only one contemporary Food and Drug Administration (FDA) thrombolytic regimen is available: alteplase (tissue plasminogen activator) $100 \mathrm{mg}$ given as a continuous peripheral intravenous infusion over $2 \mathrm{~h}$.

The hypothesis that alteplase followed by anticoagulation therapy accelerates the improvement of right ventricular function and pulmonary perfusion more rapidly than anticoagulation therapy alone was tested [7]. A multicentre trial of $101 \mathrm{PE}$ patients without initial haemodynamic instability was undertaken and the patients randomized as follows: 46 to alteplase $100 \mathrm{mg}$ over $2 \mathrm{~h}$ followed by heparin and 55 to heparin alone. Most were enrolled on the basis of high-probability lung scans. Only $20 \%$ of the patients underwent diagnostic pulmonary angiography, thus minimizing bleeding complications from groin haematomas (at the femoral vein puncture site used for angiography) among patients who received thrombolytic therapy.

No clinical episodes of recurrent PE occurred among the alteplase patients, but there were five (two fatal and three nonfatal) clinically suspected recurrent PEs within 14 days in patients randomized to heparin alone $(p=0.06)$. All five presented initially with right ventricular hypokinesis on echocardiography, despite a normal systemic arterial pressure at baseline. Thus, echocardiography identified a subgroup of patients with impending right ventricular failure at high risk of adverse outcomes if treated with heparin alone. Such patients, in particular, may be excellent candidates for aggressive intervention.

It was subsequently shown quantitatively that most patients who receive thrombolytic therapy for PE achieve recovery of regional as well as global right ventricular function [8]. At baseline, right ventricular areas were significantly larger than normal at enddiastole and end-systole. Diastolic and systolic right ventricular areas decreased after thrombolytic therapy. The area of the right ventricle most severely affected (and most improved after therapy) was the mid-right ventricular free wall.

\section{Thrombolytic therapy: bleeding complications}

Catastrophic bleeding can occur with thrombolytic therapy, regardless of specific drug or specific indication. Therefore, all patients being considered for any thrombolytic regimen should be subjected to a meticulous evaluation for possible contraindications such as intracranial disease, recent surgery or trauma. Randomized controlled trials tend to underestimate bleeding complication rates, because these trials usually have multiple safeguards to ensure appropriate exclusion of poor candidates for thrombolytic therapy. However, registries that record "real world" results, including bleeding complications, may be more realistic with respect to the limitations and problems of thrombolytic therapy.

In a series of 312 patients receiving thrombolytic therapy for PE in five clinical trials, there was a 1.9\% risk $(95 \%$ confidence interval $0.7-4.1 \%)$ of intracranial bleeding [9]. Two of the six patients received thrombolytic therapy in violation of the trial protocol, because they had known pre-existing intracranial disease. Diastolic blood pressure on admission was significantly elevated in patients who developed intracranial haemorrhage compared with those who did not (90.3 versus $77.6 \mathrm{mmHg}$; $=0.04)$. No patient under the age of $55 \mathrm{yrs}$ was affected. These data reinforce the message that meticulous patient screening before administering thrombolytic therapy is imperative.

In carefully conducted observational studies, thrombolytic therapy for PE emerges as a strategy with a higher risk of major haemorrhagic complications than is reported in controlled clinical trials. At the Laennec Hospital in Paris, France, 132 consecutive patients received alteplase for massive PE [10]. Two (1.5\%) suffered intracranial bleeding, and one of the two died. Pericardial tamponade was equally problematic and occurred in two $(1.5 \%)$ other patients, one of whom died. Other major bleeding complications included two gastrointestinal haemorrhages, three cases of haemoptysis and 11 hematomas at the puncture site for pulmonary angiography.

In the ICOPER, the prospective registry of 2,454 patients with PE conducted at 52 hospitals in seven countries, 304 patients received thrombolytic therapy [1]. Three per cent of patients who received thrombolytic therapy suffered intracranial bleeding. Overall, 
$22 \%$ of those receiving thrombolytic therapy experienced major bleeding and $12 \%$ required transfusions.

In an overview of the five PE thrombolytic therapy trials conducted by the present author and colleagues [7, 11-14], the mean age of patients with major bleeding was $63 \mathrm{yrs}$, whereas that of patients with no haemorrhagic complication was 56 yrs $(\mathrm{p}=0.005)$. There was a $4 \%$ increased risk of bleeding for each additional year of age. Increasing body mass index and pulmonary angiography were also significant predictors of haemorrhage [15].

Thus, to minimize bleeding risk, a fastidious history must be taken prior to administering thrombolytic therapy so that patients with prior moderate or severe hypertension, head trauma or seizures can readily be identified. The use of arterial puncture should be minimized. Finally, increasing age and a high body mass index should be recognized as risk factors for bleeding and should be factored into the decision regarding thrombolytic therapy versus the alternative intervention of embolectomy.

\section{Embolectomy}

Embolectomy can be undertaken in the interventional angiography laboratory or in the operating room. Catheter embolectomy is hindered by devices that are designed normally to remove small arterial clots rather than decompress massive PE. Available strategies for catheter embolectomy include fragmentation, rheolysis and aspiration [16]. For example, the Hydrolyser $\mathbb{R}$ device (Cordis Corporation, Miami Lakes, FL, USA) for massive PE consists of a $135-\mathrm{cm}$ long 6-7 $\mathrm{F}$ double lumen catheter with a closed atraumatic tip and a distal 6-mm oval side hole [17]. Saline is injected through the narrow lumen using a mechanical injector. The narrow lumen ends in a hairpin loop at the distal end of the catheter, near the side hole. As the high-speed retrograde fluid jet crosses the side hole, the resulting Venturi effect fragments the adjacent thrombus and sucks the debris into the larger evacuation lumen.

Because of the limitations of catheter embolectomy, the Collaborative Pulmonary Embolism Management Team at Brigham and Women's Hospital has shifted the emphasis of mechanical intervention towards open surgical embolectomy. This approach is usually successful if undertaken in patients who enter the operating room with stable haemodynamics [18]. In $<2$ yrs, operations have been performed on 26 patients, with a
$92 \% 30$-day survival rate. The operation is performed with a median sternotomy, continuous transoesophageal echocardiographic monitoring and cardiopulmonary bypass with a warm beating heart. Using this approach, the cardiac surgeons can visualize all lobar and most segmental pulmonary arteries.

\section{Inferior vena caval filters}

There are two indications for insertion of an inferior vena caval filter: 1) massive bleeding that precludes anticoagulation therapy; and 2) recurrent PE despite prolonged intensive anticoagulation therapy. It is important to acknowledge that filters do not halt the thrombotic process. Furthermore, they are associated with a marked increase in the frequency of subsequent DVT [19, 20].

\section{Optimal anticoagulant regimen}

Low-molecular-weight heparins offer high and sustained plasma antithrombin activity when administered in therapeutic doses, with only moderate prolongation of the activated partial thromboplastin time [21]. Low-molecular-weight heparins have pharmacokinetic advantages over unfractionated heparin [22], as detailed in table 4.

The FDA has approved two low-molecular-weight heparins for outpatient treatment of acute DVT (without PE) as a bridge to warfarin. However, neither the strategy of using a low-molecular-weight heparin nor an abbreviated length of hospital stay has been approved for patients presenting with symptomatic PE.

The largest trial of low-molecular-weight heparin for patients with symptomatic acute PE was conducted in France [23]. Overall, 612 patients were randomly assigned to once daily tinzaparin or to adjusted-dose intravenous unfractionated heparin. These heparin regimens were given as a bridge to a stable and therapeutic dose of oral anticoagulant. During the 90 days of follow-up, the complication rates were similar in the two groups. Importantly, those patients assigned to tinzaparin remained hospitalized for a mean of 7 days. Therefore, the strategy of outpatient therapy or reduced duration of hospitalization was not tested in this trial.

Oral anticoagulation therapy with warfarin remains unsatisfactory. There is a high rate of major bleeding despite optimal attempts at dose adjustment using the

Table 4.-Mechanisms responsible for the pharmacokinetic advantages of low-molecular-weight heparins over unfractionated heparin

\begin{tabular}{|c|c|}
\hline Advantage & Mechanism \\
\hline $\begin{array}{l}\text { More predictable anticoagulant } \\
\text { response }\end{array}$ & $\begin{array}{l}\text { Less binding to plasma proteins and to proteins released } \\
\text { from activated platelets and endothelial cells }\end{array}$ \\
\hline Better bioavailability at low doses & Less binding to endothelium \\
\hline Dose-independent clearance mechanism & Less binding to macrophages \\
\hline Longer half-life & Less binding to macrophages \\
\hline
\end{tabular}

(From [22].). 
International Normalized Ratio. In the Anticoagulation Service at Brigham and Women's Hospital, there were 10 patients with major bleeding out of 676 who were followed prospectively over a 2 -yr period. Of the ten patients, two bled while their prothrombin times were within the target range and three bled with subtherapeutic prothrombin times.

Potential replacements for warfarin include oral direct thrombin inhibitors. Ximelagatran, which has a molecular weight of $474 \mathrm{Da}$, is an oral prodrug that is rapidly converted to melagatran, a direct thrombin inhibitor. Ximelagatran can achieve a therapeutic anticoagulant effect with fixed twice daily dosing, without dose adjustment. This drug is currently being studied in multiple phase III trials. It appears to have a wide "therapeutic window", predictable pharmacokinetics and minimal drug/drug and food/ drug interactions.

\section{Optimal duration of anticoagulation therapy}

The optimal duration of anticoagulation therapy after acute PE remains extremely controversial, especially after idiopathic PE that occurs without relation to cancer, surgery or trauma. Schulman et al. [24] found that 6 months of oral anticoagulation therapy halved the recurrence rate over the ensuing $2 \mathrm{yrs}$, compared to 6 weeks of anticoagulation therapy. Consequently, 6 months is the usual duration of anticoagulation therapy under these circumstances. However, in the provocative study of KEARON et al. [25], patients with venous thromboembolism were randomized to 3 months or 2 yrs of anticoagulation therapy. The trial was stopped early because of the markedly lower rate of recurrence in the group receiving prolonged anticoagulation therapy. After a mean follow-up period of 10 months, there were 17 recurrences among those on 3 months of anticoagulation therapy compared with one event among those on long-term anticoagulation therapy.

However, prolonged anticoagulation therapy may simply forestall but not prevent recurrent thrombosis. Furthermore, the increased bleeding with prolonged anticoagulation therapy may negate any potential benefit of fewer recurrences. In the Warfarin Optimal Duration Italian Trial (WODIT), 267 patients with idiopathic proximal DVT were randomized to either 3 or 12 months of anticoagulation therapy [26]. They were followed clinically for $\geqslant 2$ yrs. The mortality and recurrence rates were the same in both groups. The mean time to recurrence was 11 months among patients who received 3 months of anticoagulation therapy, compared with 16 months in the group that received 12 months of anticoagulation therapy. Importantly, 3\% of patients receiving 12 months of anticoagulation therapy had major nonfatal bleeding during months $4-12$ of therapy. Thus, in WODIT, extending the 3-month course of anticoagulation therapy to $1 \mathrm{yr}$ was not associated with long-term clinical benefit.

Recently, PINEDE et al. [27] reported a randomized trial of 736 patients with venous thromboembolism. For patients with temporary risk factors such as surgery or trauma and without predisposing factors for recurrence (such as thrombophilia), they found that isolated calf DVT could be safely treated with 6 weeks rather than 3 months of anticoagulation therapy. For proximal DVT, they found that 3 months of anticoagulation therapy was as effective and safe as 6 months.

It is possible that a lower intensity of anticoagulation therapy, after the initial phase of full-dose warfarin, might reduce bleeding complications associated with long-term therapy and yet maintain a low frequency of recurrent events. To test this hypothesis, the Prevention of Recurrent Venous Thromboembolism (PREVENT) trial is evaluating the efficacy of prolonged treatment with low-intensity warfarin for the prevention of recurrent events [28]. This trial is sponsored by the National Institutes of Health, Bethesda, MD, USA. Patients with a history of documented idiopathic venous thrombosis who have completed a standard course of anticoagulation therapy are being enrolled in an ongoing randomized double-blind placebo-controlled trial comparing low-intensity anticoagulation therapy, target International Normalized Ratio 1.5-2.0, to usual care without anticoagulants. Trial end points include recurrent venous thromboembolism, major bleeding and allcause mortality. To date, $>450$ patients have been enrolled. Follow-up will continue for $\geqslant 3$ yrs [29].

Despite the high rate of recurrent venous thrombosis after discontinuation of anticoagulation therapy, there are currently insufficient data to recommend indefinite warfarin therapy following an initial acute pulmonary embolism [30]. Ongoing clinical trials are testing the strategy of prolonged anticoagulation therapy, particularly with less than the standard intensity of anticoagulation. The hope is that this approach will reduce both the recurrent events and haemorrhagic risk associated with long-term anticoagulation therapy.

\section{References}

1. Goldhaber SZ, Visani L, De Rosa M. Acute pulmonary embolism: clinical outcomes in the International Cooperative Pulmonary Embolism Registry (ICOPER). Lancet 1999; 353: 1386-1389.

2. Nakamura M, Fujioka H, Yamada N, et al. Clinical characteristics of acute pulmonary thromboembolism in Japan: results of a multicenter registry in the Japanese Society of Pulmonary Embolism Research. Clin Cardiol 2001; 24: 132-138.

3. Wicki J, Perrier A, Perneger TV, Bounameaux H, Junod AF. Predicting adverse outcome in patients with acute pulmonary embolism: a risk score. Thromb Haemost 2000; 84: 548-552.

4. Grifoni S, Olivotto I, Cecchini P, et al. Short-term clinical outcome of patients with acute pulmonary embolism, normal blood pressure, and echocardiographic right ventricular dysfunction. Circulation 2000; 101: 2817-2822.

5. Meyer T, Binder L, Hruska N, Luthe H, Buchwald $\mathrm{AB}$. Cardiac troponin I elevation in acute pulmonary embolism is associated with right ventricular dysfunction. J Am Coll Cardiol 2000; 36: 1632-1636. 
6. Giannitsis E, Muller-Bardorff M, Kurowski V, et al. Independent prognostic value of cardiac troponin $\mathrm{T}$ in patients with confirmed pulmonary embolism. Circulation 2000; 102: 211-217.

7. Goldhaber SZ, Haire WD, Feldstein ML, et al. Alteplase versus heparin in acute pulmonary embolism: randomised trial assessing right ventricular function and pulmonary perfusion. Lancet 1993; 341: 507-511.

8. Nass N, McConnell MV, Goldhaber SZ, Chyu S, Solomon SD. Recovery of regional right ventricular function after thrombolysis for pulmonary embolism. Am J Cardiol 1999; 83: 804-806.

9. Kanter DS, Mikkola KM, Patel SR, Parker JA, Goldhaber SZ. Thrombolytic therapy for pulmonary embolism. Frequency of intracranial hemorrhage and associated risk factors. Chest 1997; 111: 1241-1245.

10. Meyer G, Gisselbrecht M, Diehl JL, Journois D, Sors $\mathrm{H}$. Incidence and predictors of major hemorrhagic complications from thrombolytic therapy in patients with massive pulmonary embolism. Am J Med 1998; 105: 472-477.

11. Goldhaber SZ, Vaughan DE, Markis JE, et al. Acute pulmonary embolism treated with tissue plasminogen activator. Lancet 1986; 2: 886-889.

12. Goldhaber SZ, Kessler CM, Heit J, et al. A randomized controlled trial of recombinant tissue plasminogen activator versus urokinase in the treatment of acute pulmonary embolism. Lancet 1988; 2: 293-298.

13. Goldhaber SZ, Kessler CM, Heit JA, et al. Recombinant tissue-type plasminogen activator versus a novel dosing regimen of urokinase in acute pulmonary embolism: a randomized controlled multicenter trial. J Am Coll Cardiol 1992; 20: 24-30.

14. Goldhaber SZ, Agnelli G, Levine MN, on behalf of the Bolus Alteplase Pulmonary Embolism Group. Reduced dose bolus alteplase versus conventional alteplase infusion for pulmonary embolism thrombolysis. An international multicenter randomized trial. Chest 1994; 106: 718-724.

15. Mikkola KM, Patel SR, Parker JA, Grodstein F, Goldhaber SZ. Increasing age is a major risk factor for hemorrhagic complications following pulmonary embolism thrombolysis. Am Heart J 1997; 134: 69-72.

16. Goldhaber SZ. Integration of catheter thrombectomy into our armamentarium to treat acute pulmonary embolism. Chest 1998; 114: 1237-1238.

17. Fava M, Loyola S, Huete I. Massive pulmonary embolism: treatment with the hydrolyser thrombectomy catheter. J Vasc Interv Radiol 2000; 11: 11591164.

18. Ullmann M, Hemmer W, Hannekum A. The urgent pulmonary embolectomy: mechanical resuscitation in the operating theatre determines the outcome. Thorac Cardiovasc Surg 1999; 47: 5-8.

19. Decousus H, Leizorovicz A, Parent F, et al. A clinical trial of vena caval filters in the prevention of pulmonary embolism in patients with proximal deep-vein thrombosis. Prevention du Risque d'Embolie Pulmonaire par Interruption Cave Study Group. $N$ Engl $J$ Med 1998; 338: 409-415.

20. White RH, Zhou H, Kim J, Romano PS. A population-based study of the effectiveness of inferior vena cava filter use among patients with venous thromboembolism. Arch Intern Med 2000; 160: 2033-2041.

21. Agnelli G, Iorio A, Renga C, et al. Prolonged antithrombin activity of low-molecular-weight heparins. Clinical implications for the treatment of thromboembolic diseases. Circulation 1995; 92: 2819-2824.

22. Weitz JI. Low-molecular-weight heparins. $N$ Engl J Med 1997; 337: 688-698.

23. Simonneau G, Sors H, Charbonnier B, et al. A comparison of low-molecular-weight heparin with unfractionated heparin for acute pulmonary embolism. The THESEE Study Group. Tinzaparine ou Heparine Standard: Evaluations dans 1'Embolie Pulmonaire. N Engl J Med 1997; 337: 663-669.

24. Schulman S, Rhedin AS, Lindmarker P, et al. A comparison of six weeks with six months of oral anticoagulant therapy after a first episode of venous thromboembolism. Duration of Anticoagulation Trial Study Group. N Engl J Med 1995; 332: 1661-1665.

25. Kearon C, Gent M, Hirsh J, et al. A comparison of three months of anticoagulation with extended anticoagulation for a first episode of idiopathic venous thromboembolism. N Engl J Med 1999; 340: 901-907.

26. Agnelli G, Prandoni P, Santamaria MG, et al. Three months versus one year of oral anticoagulant therapy for idiopathic deep venous thrombosis. Warfarin Optimal Duration Italian Trial Investigators. $N$ Engl J Med 2001; 345: 165-169.

27. Pinede L, Ninet J, Duhaut $\mathrm{P}$, et al. Comparison of 3 and 6 months of oral anticoagulant therapy after a first episode of proximal deep vein thrombosis or pulmonary embolism and comparison of 6 and 12 weeks of therapy after isolated calf deep vein thrombosis. Circulation 2001; 103: 2453-2460.

28. Ridker PM. Long-term, low-dose warfarin among venous thrombosis patients with and without factor $\mathrm{V}$ Leiden mutation: rationale and design for the Prevention of Recurrent Venous Thromboembolism (PREVENT) trial. Vasc Med 1998; 3: 67-73.

29. Ridker PM. Duration and intensity of anticoagulation among patients with genetic predispositions to venous thrombosis. Curr Cardiol Rep 1999; 1: 88-90.

30. Blake GJ, Ridker PM. Optimal duration of anticoagulation following venous thromboembolism among patients with and without inherited thrombophilia. In: Goldhaber SZ, Ridker PM, eds. Thrombosis and Thromboembolism. New York, Marcel Dekker, 2002; pp. 261-282. 